Pacific Journal of Mathematics

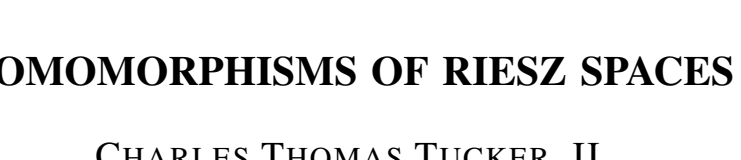




\section{HOMOMORPHISMS OF RIESZ SPACES}

\section{T. TUCKER}

If $L$ is a Riesz space (lattice ordered vector space), a Riesz homomorphism of $L$ is an order preserving linear map which preserves the finite operations " $V$ " and " $\wedge$ ". It is shown here that if $L$ is one of a large class of spaces and $\varphi$ is a Riesz homomorphism from $L$ onto an Archimedean Riesz space, then $\varphi$ preserves the order limits of sequences.

The symbol $\theta$ will be used to denote the zero element of any vector space. Suppose $L$ is a Riesz space (lattice ordered vector space). If $f \in L$ then $|f|=f \vee \theta-(f \wedge \theta)$. If $M$ is a linear subspace of $L$ then $M$ is said to be an ideal of $L$ if, whenever $|g| \leqq|f|$ and $f \in M$, then $g \in M$. If each of $L_{1}$ and $L_{2}$ is a Riesz space, a Riesz homomorphism $\varnothing$ from $L_{1}$ to $L_{2}$ is a linear map from $L_{1}$ to $L_{2}$ which preserves order and the finite operations " $V$ " and " $\Lambda$ ". A sequence $f_{1}, f_{2}, f_{3}, \cdots$ of points is said to order converge to the point $f$ if there exists a sequence $u_{1} \geqq u_{2} \geqq u_{3} \geqq \cdots$ and a sequence $v_{1} \leqq v_{2} \leqq v_{3} \leqq \cdots$ of points such that $\bigvee v_{p}=f, \wedge u_{p}=f$, and $v_{p} \leqq f_{p} \leqq u_{p}$. Order convergence for nets is defined analogously. A sequence $f_{1}, f_{2}, f_{3}, \cdots$ of elements of the Riesz space $L$ is said to converge relatively uniformly to the element $f$ of $L$ if there exists an element $g$ of $L$ (called the regulator) such that if $\varepsilon>0$, there exists a number $N_{\varepsilon}$ such that if $n$ is a positive integer greater than $N_{\varepsilon}$, then $\left|f-f_{n}\right| \leqq \varepsilon g$. A Riesz space $L$ is said to be Archimedean if, whenever $f$ and $g$ are two points of $L$ such that $0 \leqq n f \leqq g$ for all positive integers $n$, then $f=\theta$. Also $L$ is said to be $\sigma$-complete if each countable set of positive elements has a greatest lower bound and complete if each set of positive elements has a greatest lower bound. If $\phi$ is a Riesz homomorphism which preserves the order limits of sequences then $\varphi$ is said to be a Riesz $\sigma$-homomorphism. If $\varphi$ preserves the order limits of nets it is said to be a normal Riesz homomorphism. A one-to-one onto map which is a Riesz homomorphism is a Riesz isomorphism. If $H$ is a subset of $L, H^{+}$will denote the set of all points $f$ of $H$ such that $f \geqq \theta$. If $f \in L$ then $f^{+}$denotes $f \vee \theta$.

Suppose $L$ is a Riesz space, $M$ is an ideal of $L$, and the algebraic quotient $L / M$ is partially ordered as follows: If each of $H$ and $K$ belongs to $L / M$ and there is an element $h$ of $H$ and $k$ of $K$ such that $h \geqq k$, then $H \geqq K$. It follows that $L / M$ is a Riesz space and the normal map $\pi: L \rightarrow L / M$ is a Riesz homomorphism (Luxemburg and Zaanen [3], p. 102). The coset of $L / M$ containing $f$ will be denoted $[f]$. Further, if $M$ is the kernel of a Riesz homomorphism $\varphi$ defined 
on a Riesz space $L$ then the image of $\varphi$ is Riesz isomorphic to $L / M$. (Luxemburg and Zaanen [3], p. 102).

If $M$ is a subset of a Riesz space $L$ with the property that whenever $m_{1}, m_{2}, m_{3}, \cdots$ is a sequence of points of $M$ which converges relatively uniformly to a point $b$ of $L, b$ is in $M$, then $M$ is said to be uniformly closed.

In many instances properties of Riesz homomorphisms can be related to properties of their kernels. The following four theorems which are examples of this are listed for future reference.

Theorem A. If $L$ is a Riesz space and $\varphi$ is a Riesz homomorphism defined on $L$ then $\varphi(L)$ is Archimedean if and only if the kernel of $\varphi$ is uniformly closed. (See Veksler [8] or Luxemburg and Zaanen [3], Theorem 60.2.)

An ideal $M$ of $L$ is called a $\sigma$-ideal if, whenever $\left\{m_{i}\right\}$ is a countable subset of $M$ and $b=\mathrm{V} m_{i}$, then $b \in M$.

THeorem B. Suppose $L$ is a Riesz space and $\phi$ is a Riesz homomorphism from $L$ onto the Riesz space $K$. Then $\varphi$ is a Riesz $\sigma$-homomorphism if and only if the kernel of $\varphi$ is a $\sigma$-ideal. (See Luxemburg and Zaanen [3], Theorem 18.11.)

THeOREM C. Suppose $L$ is a $\sigma$-complete Riesz space and $\varphi$ is a Riesz $\sigma$-homomorphism defined on $L$. Then $\varphi(L)$ is $\sigma$-complete. (See Veksler [7] or Luxemburg and Zaanen [3], Theorem 59.3.)

An ideal $M$ of $L$ is called a band if, whenever $\left\{m_{\alpha}\right\}, \alpha \in \lambda$, is a subset of $M$ and $b=\mathrm{V} m_{\alpha}$, then $b \in M$.

Theorem D. Suppose $L$ is a Riesz space and $\varphi$ is a Riesz homomorphism from $L$ onto the Riesz space $K$. Then $\varphi$ is a normal Riesz homomorphism if and only if the kernel of $\varphi$ is a band. (See Luxemburg and Zaanen [3], Theorem 18.13.)

A question of interest is when can properties of $L$ imply properties of a class of Riesz homomorphisms defined on $L$. By combining some known results it can be noted that to place requirements on all the Riesz homomorphisms on $L$ is quite strong.

The sequence $f_{1}, f_{2}, f_{3}, \ldots$ is called a uniform Cauchy sequence (with regulator $g$ ) if, for each $\varepsilon>0$, there is a number $N$ such that if $n$ and $m$ are positive integers and $n, m>N$, then $\left|f_{n}-f_{m}\right| \leqq \varepsilon g$. The Riesz space is uniformly complete whenever every uniform Cauchy sequence (with regulator $g$ ) converges uniformly (with regulator 
g) to a point of $L$.

Proposition 1. Suppose $L$ is a uniformly complete Archimedean Riesz space. Each two of the following four statements are equivalent:

(1) For each Riesz homomorphism $\varnothing$ defined on $L, \varphi(L)$ is Archimedean,

(2) For each Riesz homomorphism $\varphi$ from $L$ onto a Riesz space $K, \varphi$ is a Riesz $\sigma$-homomorphism,

(3) For each Riesz homomorphism $\varnothing$ from $L$ onto a Riesz space $K, \varphi$ is a normal Riesz homomorphism, and

(4) There is a nonempty set $X$ such that $L$ is Riesz isomorphic to the space of all real functions which are zero except on some finite subset of $X$.

Proof. By a theorem of Luxemburg and Moore [2], (1) $\rightarrow$ (4). By Theorems A, B, and D, (4) $\rightarrow(3) \rightarrow(2) \rightarrow(1)$.

On the other hand, if requirements are placed on only a subcollection of the collection of all Riesz homomorphisms on $L$, results of wider applicability can be obtained. In particular, in the following theorems, it is shown that for a large class of Riesz spaces every Riesz homomorphism onto an Archimedean Riesz space is a Riesz $\sigma$ homomorphism.

If $\omega$ is a subset of $L, \omega^{d}$ denotes the set of all elements $g$ such that $|g| \wedge|f|=\theta$ for each point $f$ of $\omega$. If $M$ is a band in $L$ it is said to be a projection band if $L=M \oplus M^{d}$.

A principal band is a band generated by a single element. The Riesz space $L$ is said to have the principal projection property if every principal band is a projection band. The Riesz space $L$ has the principal projection property if and only if for each pair of points $f$ and $g$ of $L^{+}, V_{n=1}^{\infty}(n f \wedge g)$ exists. (See Luxemburg and Zaanan [3], Theorem 24.7.)

Order convergence in $L$ is said to be stable if whenever $f_{1}, f_{2}, f_{3}, \ldots$ is a sequence order converging to $\theta$ there is an unbounded, nondecreasing sequence of positive numbers $c_{1}, c_{2}, c_{3}, \cdots$ such that $c_{1} f_{1}$, $c_{2} f_{2}, c_{3} f_{3}, \cdots$ order converges to $\theta$. Order convergence in the spaces $L_{p}, 1 \leqq p<\infty ; l_{p}, 1 \leqq p<\infty$; and $C_{0}$ is stable.

If order convergence in $L$ is stable then every uniformly closed ideal in $L$ is a $\sigma$-ideal. Thus if $\varphi$ is a Riesz homomorphism from $L$ onto an Archimedean Riesz space $K$, then $\varphi$ is a Riesz $\sigma$-homomorphism.

For certain sets $X$ order convergence in $R^{X}$ is not stable. This can be seen as follows: Let $X$ be the set to which $x$ belongs only if $x$ is an unbounded, nondecreasing sequence of positive numbers. Let 
$f_{n}$ be the function defined on $X$ such that if $c_{1}, c_{2}, c_{3}, \cdots$ is a point of $X$ then $f_{n}\left(c_{1}, c_{2}, c_{3}, \cdots\right)$ is $1 / c_{n}$. Then $f_{1}, f_{2}, f_{3}, \cdots$ order converges to $\theta$, but if $c_{1}, c_{2}, c_{3}, \cdots$ is an unbounded, nondecreasing sequence of positive numbers then $c_{1} f_{1}, c_{2} f_{2}, c_{3} f_{3}, \cdots$ does not order converge to $\theta$ since $c_{n} f_{n}\left(c_{1}, c_{2}, c_{3}, \cdots\right)=1$ for each positive integer $n$. If $X$ is made of larger cardinality then clearly order convergence in $R^{X}$ still fails to be stable.

The author, in a paper concerned with the order properties of convergence of Baire functions [6], defined a positive element $x$ of a Riesz space $L$ to have property $c$ if for each sequence $h_{1} \leqq h_{2} \leqq$ $h_{3} \leqq \cdots$ of elements of $L$ such that $x=\bigvee h_{i}$, there exists an element $b$ of $L$ such that for each positive integer $n, b \leqq \sum_{i=1}^{n} h_{i}$.

EXAMPLE 2. The constant function 1 in $R^{x}$ has property $c$. The constant function 1 in $B[0,1]$ (the space of all Baire functions on the interval $[0,1])$ has property $c$.

Let $\omega$ be the set of all functions defined on the interval $[0,1]$ whose ranges are a subset of the rational numbers and let $Q$ be the vector space generated by $\omega$. Then $Q$ is a Riesz space with the principal projection property but is not uniformly complete. This can be seen as follows: If $f$ is in $\omega, H$ is a subset of the interval $[0,1]$, and $\widetilde{f}$ is the function obtained by setting $f$ to zero on $H$ and leaving it unchanged off $H$, then $\widetilde{f}$ is in $\omega$. For $Q$ to be a Riesz space it is sufficient that $f \vee \theta$ exists for each point $f$ of $Q$. Thus, if $f$ is in $Q$ it is of the form $\sum_{i=1}^{n} c_{i} f_{i}$ where the $f_{i}$ 's are in $\omega$. Let $H$ be the set of numbers $x$ for which $f(x)<0$. Then $f \vee \theta=\sum_{i=1}^{n} c_{i} \tilde{f}_{i}$ and $f \vee \theta$ is in $Q$. Clearly $Q$ has the principal projection property. Each point of $Q$ has as range a countable number set, but a function which fails to have this property, say $g(x)=x$ on the interval $[0,1]$, is the uniform limit of a sequence of points of $Q$. Further the constant function 1 in $Q$ has property $c$.

Let $L$ be a Riesz space and $x$ a positive element of $L$ which has property $c$ and $M$ be a sub Riesz space of $L$ containing $x$ with the property that if $f$ belongs to $L$ then there is a point $g$ to $M$ such that $g \geqq f$. Then $x$ has property $c$ in $M$.

THEOREm 3. Suppose $L$ is an Archimedean Riesz space containing a point $x$ which has property $c$. Then each Riesz homomorphism $\varphi$ of $L$ into an Archimedean Riesz space $K$ is a Riesz $\sigma$-homomorphism.

Proof. If it can be shown that $f_{1} \leqq f_{2} \leqq f_{3} \leqq \cdots \leqq \theta$ and $\bigvee f_{p}=\theta$ implies $\mathrm{V} \varphi\left(f_{p}\right)=\theta$, then the theorem is proved.

Now 


$$
\begin{aligned}
& f_{p} \vee(-x)+f_{p} \wedge(-x)=f_{p}-x \\
& \varphi\left(f_{p} \vee(-x)\right)+\varphi\left(f_{p} \wedge(-x)\right)=\varphi\left(f_{p}\right)-\varphi(x) \\
& \varphi\left(f_{p} \wedge(-x)\right)+\varphi(x)=\varphi\left(f_{p}\right)-\varphi\left(f_{p} \vee(-x)\right) \\
& \quad=\varphi\left(f_{p} \wedge(-x)+x\right)=\varphi\left(\left(f_{p}+x\right) \wedge \theta\right) \\
& \sum_{p=1}^{n} \varphi\left(\left(f_{p}+x\right) \wedge \theta\right)=\sum_{p=1}^{n} \varphi\left(f_{p}\right)-\varphi\left(f_{p} \vee(-x)\right) \\
& \varphi\left(\sum_{p=1}^{n}\left(f_{p}+x\right) \wedge \theta\right)=\sum_{p=1}^{n} \varphi\left(f_{p}\right)-\varphi\left(f_{p} \vee(-x)\right) .
\end{aligned}
$$

As $x$ has property $c$ there exists an element $b$ such that $b \leqq$ $\sum_{p=1}^{n}\left(f_{p}+x\right) \wedge \theta$ for each positive integer $n$. Thus,

$$
\varphi(b) \leqq \varphi\left(\sum_{p=1}^{n}\left(f_{p}+x\right) \wedge \theta\right)=\sum_{p=1}^{n} \varphi\left(f_{p}\right)-\varphi\left(f_{p} \vee(-x)\right) .
$$

Suppose that $u \leqq \theta$ is an upper bound for $\left\{\varphi\left(f_{p}\right)\right\}$. Then

$$
\varphi(b) \leqq \sum_{p=1}^{n}\left(u-\varphi\left(f_{p} \vee(-x)\right)\right) \leqq \sum_{p=1}^{n}(u-\varphi(-x))=n(u-\varphi(-x)) .
$$

Thus, $u-\varphi(-x) \geqq \theta$ as $K$ is Archimedean and $u \geqq \varphi(-x)$.

But if $x$ has property $c,(1 / n) x$ has property $c$ for each positive integer $n$. Therefore, $u \geqq(1 / n) \varphi(-x)$ and $u=\theta$ as $K$ is Archimedean. So $\mathbf{V} \varphi\left(f_{p}\right)=\theta$ and $\varphi$ is a Riesz $\sigma$-homomorphism.

Frequently inclusion maps do not preserve the order limits of sequences. For instance the inclusion map of the space of continuous functions on the interval $[0,1]$ into the space of all functions on the interval $[0,1]$ fails to preserve the order limits of sequences. For this reason most theorems which guarantee that a Riesz homomorphism is a Riesz $\sigma$-homomorphism require that the mappings be onto. Theorem $B$ would not be true if $\varphi$ was not specified to be an onto map because of the example just noted. However in view of Theorem 3, no such problem can arise in a space that contains an element with property $c$. Any embedding of such a space into an Archimedean space must preserve the order limits of sequences.

If in Theorem 3, $x$ is assumed to be a strong unit (a point with the property that if $f \in L$ there is a number $r$ such that $r x \geqq|f|$ ) rather than have property $c$, then the statement is no longer true. For instance, let $L$ consist of the set of all bounded sequences and $M$ be the set of all sequences $s_{1}, s_{2}, s_{3}, \cdots$ with the property that if $\varepsilon>0$ there is only a finite number of positive integers $n$ such that $\left|s_{n}\right|>\varepsilon$. Then $M$ is a uniformly closed ideal but not a $\sigma$-ideal.

The Riesz space $L$ is $\sigma$-complete if and only if it is uniformly complete and has the principal projection property (Luxemburg and Zaanan [3], Theorem 42.5). If $L$ is uniformly complete and $\varphi$ is a 
Riesz homomorphism defined on $L$ then $\varphi(L)$ is uniformly complete (Luxemburg and Moore [2]).

Thus the question of when the operation of taking a quotient preserves the property of $\sigma$-completeness can be included in the question of when this operation preserves the principal projection property.

The Riesz space $L$ has the quasi principal projection property if for each point $f$ of $L, L=\{f\}^{d} \oplus\{f\}^{d d}$. Then $L$ has the principal projection property if and only if it has the quasi principal projection property and is Archimedean. If $L$ has the quasi principal projection property then for each point $f$ of $L$ and $g$ of $L$ there is a unique element $g_{1}$ of $\{f\}^{d}$ and a unique element $g_{2}$ of $\{f\}^{d d}$ such that $g=$ $g_{1}+g_{2}$. Denote $g_{2}$ by $P_{f}(g)$.

THEOREM 4. Suppose L is a Riesz space with the quasi principal projection property, $M$ is an ideal of $L$, and $\pi$ is the natural map of $L$ onto $L / M$. Then the following two conditions are equivalent:

(1) If $m$ is a point of $M, P_{m} L$ is a subset of $M$ and

(2) (a) $L / M$ has the quasi principal projection property and

(b) $\pi P_{f}=P_{\pi f} \pi$ for each point $f$ of $L$.

Proof. Suppose Condition 1 is true and each of $H$ and $K$ belongs to $(L / M)^{+}$. We wish to show that there exist points $H_{1}$ and $H_{2}$ belonging to $K^{d}$ and $K^{d d}$ respectively such that $H=H_{1}+H_{2}$. There exist points $h$ and $k$ in $L^{+}$such that $H=[h]$ and $K=[k]$. As $L$ has the quasi principal projection property there exist points $h_{1}$ and $h_{2}$ of $\{k\}^{d}$ and $\{k\}^{d d}$ respectively such that $h=h_{1}+h_{2}$. Now $H=\left[h_{1}\right]+\left[h_{2}\right]$ and $\left[h_{1}\right] \wedge\left[h_{2}\right]=\theta$. Since $h_{1}$ is in $\{k\}^{d}, h_{1} \wedge k=\theta$, so $\left[h_{1}\right] \wedge[k]=$ $\left[h_{1} \wedge k\right]=\theta$ and $\left[h_{1}\right]$ belongs to $\{K\}^{d}$. Suppose $J \geqq \theta$ is in $\{K\}^{d}$, i.e., $J \wedge K=\theta$. There is a point $j$ of $L^{+}$such that $[j]=J$. There is a point $m$ of $M$ such that $j \wedge k=m$. By hypothesis there exists a point $m_{1}$ of $M$ such that $P_{m}(j)=m_{1}$. Thus there is a point $j_{1} \geqq \theta$ and a point $m_{1} \geqq \theta$ such that $j_{1}+m_{1}=j, j_{1}$ is in $\{j \wedge k\}^{d}$, and $m_{1}$ is in $\{j \wedge k\}^{d d}$. Since $j_{1}+m_{1}=j$ and $m_{1} \geqq \theta, j_{1} \leqq j$ and $j_{1} \wedge j=j_{1}$. Therefore, $\theta=j_{1} \wedge(j \wedge k)=\left(j_{1} \wedge j\right) \wedge k=j_{1} \wedge k$ or $\left(j-m_{1}\right) \wedge k=$ $\theta$. So $j-m_{1}$ is in $\{k\}^{d}$ and hence $\left(j-m_{1}\right) \wedge h_{2}=\theta$. It follows that $[j] \wedge\left[h_{2}\right]=\theta$ and $\left[h_{2}\right]$ is in $\{K\}^{d d}$.

Also $\pi P_{k}(h)=\pi\left(h_{2}\right)=\left[h_{2}\right]=P_{K}(H)=P_{\pi k} \pi(h)$.

Suppose Condition 2 is true. If $m$ is a point of $M$ and $h$ is a point of $L$

$$
\theta=P_{\theta} \pi(h)=P_{\pi m} \pi(h)=\pi P_{m}(h) .
$$

Thus $P_{m}(h)$ belongs to $M$.

COROLLARY 5. Suppose $L$ is a Riesz space with the quasi 
principal projection property, $M$ is an ideal of $L$, and $\pi$ is the natural map of $L$ onto $L / M$. Then the following two conditions are equivalent:

(1) (a) If $m$ is a point of $M, P_{m} L$ is a subset of $M$ and

(b) $M$ is relatively uniformly closed, and

(2) (a) $L / M$ has the principal projection property and

(b) $\pi P_{f}=P_{\pi f} \pi$ for each point $f$ of $L$.

Proof. For $L / M$ to have the principal projection property it is equivalent that $L / M$ have the quasi principal projection property and be Archimedean. By Theorem $\mathrm{A}$ it is necessary and sufficient for $L / M$ to be Archimedean that $M$ be uniformly closed.

TheORem 6. Suppose L is a Riesz space with the quasi principal projection property and $M$ is an ideal of $L$. Consider the following two properties:

(1) (a) If $m$ is a point of $M, P_{m} L$ is a subset of $M$ and

(b) $M$ is relatively uniformly closed, and

(2) $M$ is a $\sigma$-ideal.

Then properties 1 and 2 are independent. If $L$ is assumed to have the principal projection property then property 2 implies property 1 but property 1 does not necessarily imply property 2. If $L$ is assumed to be uniformly complete then property 1 implies property 2 , but property 2 does not necessarily imply property 1.

Proof. Suppose $L$ is assumed to have the principal projection property and property 2. For each positive integer $n$ and point $m$ of $M, n m \wedge h$ belongs to $M$ as $M$ is an ideal. Now $P_{m} h=\mathrm{V}(n m \wedge h)$, $P_{m} h$ belongs to $M$ since $M$ is a $\sigma$-ideal, and property 1 (a) holds. Property $1(\mathrm{~b})$ is clearly true.

An example of a space with the principal projection property in which property 1 does not imply property 2 is the following: Let $L$ be the subspace of the space of all sequences generated by the collection of all constant sequences and all sequences which are zero except for a finite number of terms. Let $M$ be the ideal consisting of the collection of all sequences which are zero except for a finite number of terms. Then $M$ satisfies property 1 but not property 2 .

Assume $L$ is uniformly complete and property 1 is true. Suppose $\left\{m_{1}, m_{2}, m_{3}, \cdots\right\}$ is a subset of $M^{+}$and $h=\mathbf{V}_{i=1}^{\infty} m_{i}$. Let $r_{p}=\mathbf{V}_{i=1}^{p} m_{i}$. Then $\theta \leqq r_{1} \leqq r_{2} \leqq r_{3} \leqq \cdots$ and $\bigvee_{i=1}^{\infty} r_{i}=h$. Let $j$ be a positive integer, $f_{1}=P_{r_{j+1}} h, f_{2}=h-f_{1}, g_{1}=P_{r_{j}} h, g_{2}=h-g_{1}$, and $d_{j}=f_{1}-g_{1}$. Note that $d_{j}$ is in $M$. Since $f_{1}+f_{2}=g_{1}+g_{2}, d_{j}=g_{2}-f_{2}$. As each of $g_{2}$ and $f_{2}$ is in $\left\{r_{j}\right\}^{d}, d_{j}$ is in $\left\{r_{j}\right\}^{d}$ and $d_{j} \wedge g_{1}=\theta$. Thus $d_{j} \vee g_{1}=f_{1}$. Therefore, there exists a countable pairwise disjoint subset $\left\{d_{1}, d_{2}\right.$, 
$\left.d_{3}, \cdots\right\}$ of $M$ such that $h=\mathbf{V}_{i=1}^{\infty} d_{i}$. Now the sequence $d_{1}, d_{1}+(1 / 2) d_{2}$, $d_{1}+(1 / 2) d_{2}+(1 / 3) d_{3}, d_{1}+(1 / 2) d_{2}+(1 / 3) d_{3}+(1 / 4) d_{4}, \cdots$ converges relatively uniformly to a point $m$ of $M$. Then $h$ belongs to the band generated by $m, P_{m} h=h$, and it follows that $h$ is in $M$.

An example of a uniformly complete space with the quasi principal projection property in which property 2 does not imply property 1 is the lexiographically ordered plane. The vertical axis is a $\sigma$-ideal but does not have property 1 (a).

Suppose $L$ is a Riesz space and $e \geqq \theta$ is a point of $L$. Then $e$ will be called a weak unit if $e \wedge|f|=\theta$ only in case $f=\theta$.

When necessary, it will be assumed that $L$ is a subspace of the set of all almost finite extended real valued continuous functions on an extremally disconnected compact Hausdorff space $S$. Further if $L$ has a weak unit $e$, this subspace may be chosen so that $e$ is the function identically to 1 .

Suppose $e$ is a weak unit of the Riesz space $L$. The pair $(L, e)$ will be said to be a Vulikh algebra if a multiplication can be defined on $L$ which makes it an associative, commutative algebra with multiplicative unit $e$ which is positive in the sense that if $f \geqq \theta$ and $g \geqq \theta$ then $f g \geqq \theta$. For some properties of Vulikh algebras see Rice [4], Tucker [5], or Vulikh [9], [10].

Suppose that it is assumed that $L$ is a subspace of the set of all almost finite extended real valued continuous functions on an extremally disconnected compact Hausdorff space $S$ and that $e$ is the function identically equal to 1 . If each of $f$ and $g$ belong to $L$ their pointwise product will be defined as follows: Both $f$ and $g$ are finite on a dense subset $Q$ of $S$. Their pointwise product on $Q$ is a continuous function on $Q$ and can be extended uniquely to a continuous function on $S$, since $S$ is extremally disconnected.

There is at most one multiplication which makes $(L, e)$ a Vulikh algebra (Kantorovitch, Vulikh, and Pinsker [1]). If $(L, e)$ is a Vulikh algebra and it is represented as a Riesz space as a subspace of the set of all almost finite extended real valued continuous functions on an extremally disconnected compact Hausdorff space with $e$ the constant function 1, then the Vulikh algebra multiplication will be the same as the pointwise multiplication described above.

TheORem 7. Suppose $L$ is a Riesz space with the principal projection property, $M$ is a uniformly closed ideal of $L, \pi$ is the natural map of $L$ onto $L / M$ and for each $m$ in $M^{+}$, if $K$ is the principal band generated by $m,(K, m)$ is a Vulikh algebra. Then $L / M$ has the principal projection property and $\pi P_{f}=P_{\pi f} \pi$ for each point $f$ of $L$. 
Proof. By Theorem 4 it is sufficient to show that for each point $m$ of $M^{+}$and $f$ of $L^{+}$that $\bigvee(n m \wedge f)$ belongs to $M$. Let $K$ be the principal band generated by $m$.

By the representation theorem for Riesz spaces $K$ can be assumed to consist of almost finite continuous extended real valued functions on a compact Hausdorff space $S$, where $m$ is the constant function with value 1 everywhere.

Let $h=\mathrm{V}(n m \wedge f)$. The point $h$ belongs to $K$. By hypothesis $(K, m)$ is a Vulikh algebra. Thus $h^{2}$ belongs to $K$.

Suppose $x$ is a point of $S$. If $h(x) \geqq n$, then

$$
(h-(n m \wedge f))(x) \leqq h(x) \leqq \frac{1}{n} h^{2}(x) .
$$

If $h(x)<n$, then

$$
(h-(n m \wedge f))(x)=0 \leqq \frac{1}{n} h^{2}(x) .
$$

Thus $m \wedge f, 2 m \wedge f, 3 m \wedge f, \cdots$ converges relatively uniformly to $h$ with regulator $h^{2}$. As $M$ is uniformly closed, $h$ is in $M$.

If $\alpha$ is a subset of $L^{+}$with the property that for each two points $f$ and $g$ of $\alpha, f \wedge g=\theta$, then $\alpha$ is said to be orthogonal.

THEOREM 8. Suppose $L$ is a Riesz space with the principal projection property, $M$ is a uniformly closed ideal of $L$ with the property that if $\left\{f_{1}, f_{2}, f_{3}, \cdots\right\}$ is a bounded countable orthogonal subset of $M^{+}$there is an unbounded nondecreasing positive number sequence $c_{1}, c_{2}, c_{3}, \cdots$ such that $\left\{c_{1} f_{1}, c_{2} f_{2}, c_{3} f_{3}, \cdots\right\}$ is bounded, and $\pi$ is the natural map of $L$ onto $L / M$. Then $L / M$ has the principal projection property and $\pi P_{f}=P_{\pi f} \pi$ for each point $f$ of $L$.

Proof. By Theorem 4 it is sufficient to show that for each point $m$ of $M^{+}$and $f$ of $L^{+}$that $\mathrm{V}(n m \wedge f)$ belongs to $M$.

Let $K$ be the principal band generated by $m$. By hypothesis $K$ is a projection band, let $h=\mathrm{V}(n m \wedge f)$. The point $h$ belongs to $K$. Also $\mathrm{V}(n m \wedge f)=\mathrm{V}(n m \wedge h)$.

If $k$ is in $K^{+}$, let $\chi(k)=\mathbf{V}(n k \wedge m)$. This supremum exists as $K$ has the principal projection property. Let

$$
d_{n}=\chi\left((n m \wedge h-(n-1) m)^{+}\right)-\chi\left(((n+1) m \wedge h-n m)^{+}\right) .
$$

By the representation theorem for Riesz spaces $K$ can be assumed to consist of almost finite continuous extended real valued functions on a compact Hausdorff space $S$, where $m$ is the constant function with value 1 everywhere.

Suppose $x$ is a point of $S$. If $h(x)>n$, then $d_{n}(x)=0$, if 
$n \geqq h(x)>n-1$, then $d_{n}(x)=1$, and if $h(x) \leqq n-1$, then $d_{n}(x)=0$. Let $h_{n}=(n m \wedge h-(n-1) m)^{+}-\chi\left((h-n m)^{+}\right)+(n-1) d_{n}$. If $h(x)>n$, then $h_{n}(x)=0$, if $n \geqq h(x)>n-1$, then $h_{n}(x)=h(x)$, and if $h(x) \leqq$ $n-1$, then $h_{n}(x)=0$.

Therefore $\left\{h_{1}, h_{2}, h_{3}, \cdots\right\}$ is an orthogonal subset of $M^{+}$bounded above by $h$. By hypothesis there is an unbounded nondecreasing positive number sequence $c_{1}, c_{2}, c_{3}, \cdots$ such that $\left\{c_{1} h_{1}, c_{2} h_{2}, c_{3} h_{3}, \cdots\right\}$ is bounded above by a point $b$ of $L$. Then if $i$ is a positive integer, $h-\left(h_{1}+h_{2}+\cdots+h_{i}\right) \leqq\left(1 / c_{i+1}\right) b$, and the sequence $h_{1}, h_{1}+h_{2}, h_{1}+$ $h_{2}+h_{3}, \cdots$ converges relatively uniformly to $h$. As $M$ is uniformly closed, $h$ is in $M$.

Corollary 9. Suppose $L$ is a Riesz space which is $\sigma$-complete and with the property that if $\left\{f_{1}, f_{2}, f_{3}, \cdots\right\}$ is a bounded countable orthogonal subset of $L^{+}$there is an unbounded nondecreasing positive number sequence $c_{1}, c_{2}, c_{3}, \cdots$ such that $\left\{c_{1} f_{1}, c_{2} f_{2}, c_{3} f_{3}, \cdots\right\}$ is bounded then every Riesz homomorphism $\varphi$ from $L$ onto an Archimedean Riesz space is a Riesz $\sigma$-homomorphism.

EXAMPLe 10. Suppose $L$ is one of the space $L_{p}, 1 \leqq p<\infty ; l_{p}$, $1 \leqq p<\infty$; or $C_{0}$ in which order convergence is stable or $L$ is one of the spaces $R^{X}$ or $B[0,1]$ which has a point with property $c$ as described in Example 2. Then $L$ satisfies the conditions of Corollary 9. On the other hand, let $L$ be the space of all functions defined on the $x$ axis with compact support. In this case $L$ satisfies the hypothesis of Corollary 9, while $L$ neither contains a point with property $c$ nor is order convergence stable in $L$.

By what has just been shown, if $L$ is a $\sigma$-complete Riesz space with the property that if $\left\{f_{1}, f_{2}, f_{3}, \cdots\right\}$ is a bounded countable orthogonal subset of $L^{+}$then there is an unbounded nondecreasing positive number sequence $c_{1}, c_{2}, c_{3}, \cdots$ such that $\left\{c_{1} f_{1}, c_{2} f_{2}, c_{3} f_{3}, \cdots\right\}$ is bounded is sufficient to imply that every uniformly closed ideal is a $\sigma$-ideal, but this condition is not necessary, as the following example shows.

EXAMPLE 11. Let $S$ be the set of all ordered pairs of positive integers. Let $L$ be the collection to which $f$ belongs only in case $f$ is a real valued function on $S$ with the property that there is a set $\omega$ which includes all but at most a finite number of positive integers such that if $k$ is a positive integer in $\omega, f(1, k), f(2, k), f(3, k), \cdots$ is a bounded number sequence.

The space $L$ is a complete Riesz space.

Suppose $M$ is an ideal which is uniformly closed. Let $f$ be the l.u.b. of a countable subset $\alpha$ of $M$. Let $\beta$ be the collection to which 
$g$ belongs only in case there is a positive integer $k$ and a member $h$ of $\alpha$ such that $g(k, p)=h(k, p)$ for each positive integer $p$ and if $i$ is a positive integer not $k$ then $g(i, p)=0$ for each positive integer $p$. Then $f$ is the l.u.b. of $\beta$. For each positive integer $k$, let $f_{k}$ be the function such that $f_{k}(k, p)=f(k, p)$ for each positive integer $p$ and if $i$ is a positive integer not $k$ then $f_{k}(i, p)=0$ for each positive integer $p$.

The function which is equal to $f(i, j)$ at $(i, j)$ and zero elsewhere is in $M$. Then since the function which is $p f_{k}(i, p)$ at $(i, p)$ is in $L$, $f_{k}$ is in $M$. Since the function which is if $(i, j)$ at $(i, j)$ is in $L, f$ is in $M$.

Thus each uniformly closed ideal of $M$ is a $\sigma$-ideal. For each positive integer $i$ let $g_{i}$ be the function such that $g_{i}(p, q)=1$ if $p=i$ and $g_{2}(p, q)=0$ if $i \neq p$. Then $\left\{g_{1}, g_{2}, g_{3}, \cdots\right\}$ is an orthogonal subset of $L$ which is bounded above by the constant function 1 but there is no nondecreasing unbounded positive number sequence $c_{1}, c_{2}, c_{3}, \cdots$ such that $\left\{c_{1} g_{1}, c_{2} g_{2}, c_{3} g_{3}, \cdots\right\}$ is bounded above.

The Riesz space $L$ has the projection property if every band in $L$ is a projection band. Suppose $L$ has the projection property, $\omega$ is a subset of $L, H$ is the band generated by $\omega$, and $f$ is a point of $L$. There is a unique point $f_{1}$ of $H^{d}$ and a unique point $f_{2}$ of $H$ such that $f=f_{1}+f_{2}$. Denote $f_{2}$ by $P_{\omega}(f)$.

The analogous question of when can the projection property be preserved in a natural manner can be answered easily.

THEOREM 12. Suppose $L$ is a Riesz space with the projection property, $M$ is an ideal of $L$, and $\pi$ is the natural map of $L$ onto $L / M$. Then the following two properties are equivalent:

(1) $\pi$ is a normal Riesz homomorphism, and

(2) (a) $L / M$ has the projection property, and

(b) $\pi P_{\omega}=P_{\pi \omega} \pi$ for each subset $\omega$ of $L$.

Proof. If (1) is true then the kernel of $\pi, M$, is a projection band and 2 (a) and (b) clearly hold. If (2) is true and $\omega$ is a subset of $M$ with the point $f$ as least upper bound, then $\pi P_{\omega} f=\pi f$, but $P_{\pi \omega} \pi f=$ $P_{\theta} \pi f=\theta$.

Also, several answers to the question of when is every Riesz $\sigma$ homomorphism from an Archimedean Riesz space $L$ onto a Riesz space $K$ a normal Riesz homomorphism are given in Theorem 29.3 of Luxemburg and Zaanen [3]. 


\section{REFERENCES}

1. L. Kantorovitch, B. Vulikh, and A. Pinsker, Functional Analysis in Partially Ordered Spaces, Gostekhizdat, Moscow, 1950, (Russian).

2. W. A. J. Luxemburg and L. C. Moore, Jr., Archimedean quotient Riesz spaces, Duke Math. J., 34 (1967), 725-740.

3. W. A. J. Luxemburg and A. C. Zaanen, Riesz Spaces I, North-Holland, Amsterdam, 1971.

4. Norman M. Rice, Multiplication in vector lattices, Canad. J. Math., 20 (1968), 11361149 .

5. C. T. Tucker, Sequentially relatively uniformly complete Riesz spaces and Vulikh algebras, Canad. J. Math., 34 (1972), 1110-1113.

6. - On a theorem concerning Baire functions, Proc. Amer. Math. Soc., 41 (1973), 173-178.

7. A. I. Veksler, On factor lineals in vector lattices, A.I. Gercen Ped. Inst., Leningrad, 183 (1958), 107-127.

8. — The Archimedean principle in homomorphic images of l-groups and of vector lattices, 1zv. Vysš. Učebn Zaved. Mathematika 1966, 5 (53), 33-38.

9. B. Z. Vulikh, The product in linear partially ordered spaces and its applications to the theory of operators, Mat. Sb. (N.S.), 22 (64) (1948); I, 27-78; II, 267-317, (Russian). 10. - Introduction to the Theory of Partially Ordered Spaces, Wolters-Noordhoff, Groningen, 1967.

Received March 5, 1973.

UNIVERSITY OF HOUSTON 


\section{PACIFIC JOURNAL OF MATHEMATICS}

EDITORS

RICHARD ARENS (Managing Editor)

University of California

Los Angeles, Calıfornia 90024

R. A. Beaumont

University of Washington

Seattle, Washington 98105
J. DugundJI

Department of Mathematics

University of Southern California

Los Angeles, California 90007

D. Gilbarg and J. Milgram

Stanford University

Stanford, California 94305

\section{ASSOCIATE EDITORS}
E. F. BECKENBACH
B. H. NeumanN
F. WOLF
K. YOSHIDA

\section{SUPPORTING INSTITUTIONS}

UNIVERSITY OF BRITISH COLUMBIA

CALIFORNIA INSTITUTE OF TECHNOLOGY

UNIVERSITY OF CALIFORNIA

MONTANA STATE UNIVERSITY

UNIVERSITY OF NEVADA

NEW MEXICO STATE UNIVERSITY

OREGON STATE UNIVERSITY

UNIVERSITY OF OREGON

OSAKA UNIVERSITY
UNIVERSITY OF SOUTHERN CALIFORNIA

STANFORD UNIVERSITY

UNIVERSITY OF TOKYO

UNIVERSITY OF UTAH

WASHINGTON STATE UNIVERSITY

UNIVERSITY OF WASHINGTON

AMERICAN MATHEMATICAL SOCIETY NAVAL WEAPONS CENTER 


\section{Pacific Journal of Mathematics}

\section{Vol. 55, No. $1 \quad$ September, 1974}

Robert Lee Anderson, Continuous spectra of a singular symmetric

differential operator on a Hilbert space of vector-valued functions . . . $\quad 1$

Michael James Cambern, The isometries of $L^{p}(X, K) \ldots \ldots \ldots \ldots \ldots . . \ldots$

R. H. Cameron and David Arne Storvick, Two related integrals over spaces of continuous functions ................................

Gary Theodore Chartrand and Albert David Polimeni, Ramsey theory and

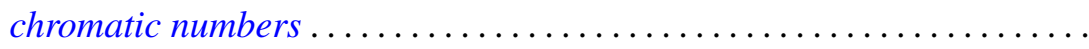

John Deryck De Pree and Harry Scott Klein, Characterization of collectively compact sets of linear operators ...................

John Deryck De Pree and Harry Scott Klein, Semi-groups and collectively compact sets of linear operators ....................... 55

George Epstein and Alfred Horn, Chain based lattices.............. 65

Paul Erdős and Ernst Gabor Straus, On the irrationality of certain series . . 85

Zdeněk Frolík, Measurable uniform spaces................... 93

Stephen Michael Gagola, Jr., Characters fully ramified over a normal

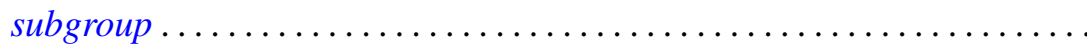

Frank Larkin Gilfeather, Operator valued roots of abelian analytic

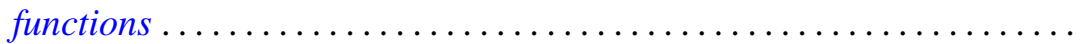

D. S. Goel, A. S. B. Holland, Cyril Nasim and B. N. Sahney, Best approximation by a saturation class of polynomial operators

James Secord Howland, Puiseux series for resonances at an embedded

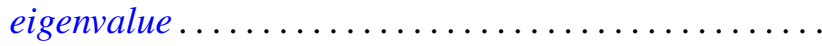

David Jacobson, Linear GCD equations .................

P. H. Karvellas, A note on compact semirings which are multiplicative

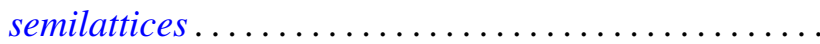

Allan Morton Krall, Stieltjes differential-boundary operators. II . .

D. G. Larman, On the inner aperture and intersections of convex sets

S. N. Mukhopadhyay, On the regularity of the $P^{n}$-integral and its application to summable trigonometric series ....... .

Dwight Webster Read, On $(J, M, m)$-extensions of Boolean algebras ....

David Francis Rearick, Multiplicativity-preserving arithmetic power series.

Indranand Sinha, Characteristic ideals in group algebras

Charles Thomas Tucker, II, Homomorphisms of Riesz spaces . . .

Kunio Yamagata, The exchange property and direct sums of indecomposable injective modules. 